\title{
Do Stress Markers and Anesthetic Technique Predict Delirium in the Elderly?
}

\author{
Stacie Deiner ${ }^{a-c}$ Hung-Mo Lin ${ }^{d}$ Daniella Bodansky ${ }^{a}$ \\ Jeffrey Silverstein ${ }^{a, c}$ Mary Sano $^{e}$ \\ Departments of a Anesthesiology, ${ }^{\mathrm{b}}$ Neurosurgery, ${ }^{\mathrm{c}}$ Geriatrics and Palliative Care, \\ ${ }^{\mathrm{d}}$ Health Evidence and Policy, and ${ }^{\mathrm{e}}$ Psychiatry, Icahn School of Medicine at Mount Sinai, \\ New York, N.Y., USA
}

\section{Key Words}

Age - Attentional dysfunction/cognition - Delirium - Outcome measures · Stress hormone ·

Surgical pathology

\begin{abstract}
Background: Postoperative delirium (PD) is a prevalent complication of elderly surgical patients, which predisposes to worsened cognitive recovery and dementia. Risk of PD has been associated with increasing magnitude of the hypothalamic-pituitary-adrenal stress response (serum cortisol, epinephrine and norepinephrine) to surgery. Anesthetics suppress this response; however, some (total intravenous anesthesia, TIVA) more than others (anesthetic gases). Prior comparisons of anesthetics have been equivocal but have not included stress markers. We hypothesized that TIVA would decrease serum stress markers and the incidence of PD. Methods: We performed a prospective cohort study of 76 elderly major surgical patients. Patients received TIVA or sevoflurane gas, and blood was drawn for serum markers pre-, intra-, and postoperatively. PD was assessed with the Confusion Assessment Method. We compared stress markers and PD between patients who received TIVA versus sevoflurane, and then modeled PD including stress and anesthetic. Results: The group that received TIVA during surgery demonstrated lower levels of all stress markers compared to the gas group, but no difference in PD. However, across groups, the postoperative norepinephrine level was much higher in patients who developed PD. Other markers and other times had no effect. Conclusion: The development of PD depends more on postoperative stress than intraoperative stress or anesthetic.


Deiner et al.: Do Stress Markers and Anesthetic Technique Predict Delirium in the Elderly?

\section{Introduction}

One of the most prevalent and morbid complications for elderly surgical patients is postoperative delirium (PD). This acute confusional state occurs hours to days after surgery in $15-53 \%$ of patients and is associated with worsened cognitive recovery after illness, longterm cognitive impairment and increased risk of dementia [1-7]. PD is responsible for longer hospital stays and greater median hospital costs estimated at 6.9 billion dollars of additional Medicare expenditures every year [8-10]. Anesthesia and choice of anesthetic medications are an intuitive culprit; however, there have been a limited number of studies comparing delirium incidence between anesthetic medications with very limited common ground between them [11]. Hence equipoise remains regarding whether delirium and other neurocognitive outcomes differ by choice of anesthetic drug [12].

Anesthetic could have a direct impact on the incidence of delirium based on the theory that PD may be a 'sickness behavior' resulting from an extreme manifestation of stress response to surgery as mediated by the hypothalamic-pituitary-adrenal axis including serum cortisol, epinephrine and norepinephrine $[13,14]$. While there are data to suggest that hyperresponsiveness of the hypothalamic-pituitary-adrenal axis is associated with PD in elderly surgical patients, there has been no direct comparison of anesthetic agents and their ability to reduce stress response and PD [15].

All anesthetics suppress stress response to surgery; however, some more than others [16]. Anesthetic gases only mildly suppress the cortisol response to surgery; but total intravenous anesthesia (TIVA) with propofol significantly blunts the cortisol response and production of catecholamines $[17,18]$. However, this information has been generalized from younger patients. The difference in stress response between anesthetic medications and delirium in the elderly has not been studied.

A randomized trial of TIVA versus gas is difficult due to the constraints of surgery (e.g. spine surgery requiring neuromonitoring). To directly investigate anesthetic choice, stress response and incidence of $\mathrm{PD}$, we performed a prospective cohort study and hypothesized that elderly surgical patients who received TIVA would have lower serum markers of stress than those receiving gas and therefore have a lower incidence of PD as measured by the Confusion Assessment Method (CAM) [19]. In post hoc analyses, we modeled the outcome of PD adjusted for both stress response and anesthetic to identify risk factors for the elderly surgical patients.

\section{Methods}

With institutional review board approval and informed consent from the patients, we performed a prospective study of patients over age 68 scheduled for major noncardiac surgery under general anesthesia, including general, spine, urologic or thoracic surgery, at the Mount Sinai Hospital, New York, N.Y., USA. Major surgery was defined as a planned hospital stay of at least 2 days. Exclusion criteria were intracranial surgery, cardiac surgery, preexisting neuropsychiatric disease, history of cerebrovascular accident (CVA, stroke) with residual deficits, baseline Mini-Mental State Exam (MMSE) score $<20$ or unable to consent to study participation, and/or unable to speak English. Eligible patients were identified using a computerized scheduling system and approached by phone at least $24 \mathrm{~h}$ prior to surgery.

Seventy-six patients consented and completed the in-hospital portion of the protocol. The medical history was obtained from the patients and confirmed by the anesthesiologist at the time of surgery. The patients were submitted to a 1-hour assessment prior to the day of surgery but within 30 days including a neuropsychological battery, the MMSE and CAM $[19,20]$. Demographics and medical history were collected from patient interviews, and the medical record (coronary artery disease, diabetes, hypertension, malignancy, renal disease, respiratory disease or history of transient ischemic attack/stroke) was confirmed by a presurgical exam completed by a physician. The American Society of Anesthesiology (ASA) classification was 
Dementia

and Geriatric

Cognitive Disorders

Table 1. Baseline comparison of the gas versus TIVA groups

\begin{tabular}{l|l}
\hline \multicolumn{2}{l|}{ Dement Geriatr Cogn Disord 2014;38:366-374 } \\
\hline DOI: 10.1159/000363762 & $\begin{array}{l}\text { @ 2014 S. Karger AG, Basel } \\
\text { www.karger.com/dem }\end{array}$ \\
\hline
\end{tabular}

Deiner et al.: Do Stress Markers and Anesthetic Technique Predict Delirium in the Elderly?

\begin{tabular}{lccr}
\hline & Gas $(\mathrm{n}=36)$ & TIVA $(\mathrm{n}=40)$ & p value \\
\hline Age, years & $76.33 \pm 5.75$ & $73.48 \pm 5.03$ & 0.024 \\
Gender & & & \\
$\quad$ Female & $21(58.3 \%)$ & $19(47.5 \%)$ & 0.345 \\
$\quad$ Male & $15(41.7 \%)$ & $21(52.5 \%)$ & \\
ASA status & & & \\
1 & $1(2.78 \%)$ & $0(0 \%)$ & \\
2 & $3(8.33 \%)$ & $15(37.5 \%)$ & 0.021 \\
3 & $26(72.22 \%)$ & $21(52.5 \%)$ & \\
$\quad 4$ & $6(16.67 \%)$ & $4(10.0 \%)$ & \\
CAD & $7(19.4 \%)$ & $5(12.5 \%)$ & 0.407 \\
CVA & $1(2.8 \%)$ & $4(10.0 \%)$ & 0.362 \\
Diabetes & $8(22.2 \%)$ & $8(20.0 \%)$ & 1.000 \\
Malignancy & $25(69.4 \%)$ & $11(28.2 \%)$ & $<0.001$ \\
Renal disease & $4(11.1 \%)$ & $3(7.5 \%)$ & 0.702 \\
Surgery & $1(2.78 \%)$ & $36(90 \%)$ & $<0.0001$ \\
$\quad$ Spine & $7(19.44 \%)$ & $2(5 \%)$ & \\
Urologic & $20(55.56 \%)$ & $2(5 \%)$ & \\
$\quad$ General & $8(22.22 \%)$ & $0(0 \%)$ & \\
Thoracic & & & \\
Preoperative & $27[26-30]$ & $29[28-30]$ & 0.029 \\
$\quad$ MMSE score & & & \\
\hline
\end{tabular}

Data are presented as mean $\pm \mathrm{SD}$, median [25th to 75 th percentile], or number (\%). The tests used for group comparisons are the 2-sample $\mathrm{t}$ test, Wilcoxon rank sum test, $\chi^{2}$ test or Fisher's exact test, as appropriate. $\mathrm{CAD}=$ Coronary artery disease , CVA = cerebrovascular accident.

used, which assesses the physical status, where ASA 1 means healthy, ASA 2 mild disease, ASA 3 systemic disease which limits function, ASA 4 disease which is a constant threat to life, and ASA 5 not expected to survive as determined by the anesthesiologist. The intraoperative data that were collected included vital signs, estimated blood loss (EBL), anesthetic, surgery type and surgery duration (table 2). On the day of surgery, patients had blood drawn for stress markers: cortisol, epinephrine and norepinephrine immediately prior to surgery, $4 \mathrm{~h}$ after surgery started, and at $2 \mathrm{~h}$ after surgery.

Patients received either TIVA consisting of propofol infusion or gas (sevoflurane). The choice of gas versus TIVA anesthetic was determined by the clinical anesthesia team based on requirements for the procedure. The anesthesia team was instructed to avoid benzodiazepines and nitrous oxide but was permitted to choose any induction agent and narcotic or paralytic agent. Patients were seen by the research team and assessed with the CAM and MMSE in the recovery room (delirium in the postoperative care unit or emergence delirium) and on each postoperative day (PD).

\section{Statistical Analysis}

The $\chi^{2}$ test or Fisher's exact test for categorical variables, and Student's t test or Wilcoxon rank sum test for continuous variables were used to compare the TIVA and gas groups at baseline, with respect to intraoperative variables and the incidence of PD. These analytic methods were also applied to the post hoc analysis to compare patients who did and did not develop PD (table 3). Variables associated with PD with a p value $<0.2$ in univariate analysis and/or of clinical significance were used to fit a forward stepwise logistic regression model of delirium which developed in the postoperative days. A receiver operating characteristic curve was generated to demonstrate prediction ability, and the Pearson goodness-of-fit test was used to show model fit. Model coefficients, odds ratios and the corresponding 95\% confidence intervals are listed in table 4 
Deiner et al.: Do Stress Markers and Anesthetic Technique Predict Delirium in the Elderly?

Table 2. Comparison of the gas versus TIVA groups

\begin{tabular}{|c|c|c|c|}
\hline & Gas $(n=36)$ & TIVA $(n=40)$ & $\mathrm{p}$ value \\
\hline Baseline MMSE score & $27[26-30]$ & $29[28-30]$ & 0.029 \\
\hline $\mathrm{EBL}, \mathrm{ml}$ & $200[100-500]$ & $375[200-750]$ & 0.029 \\
\hline Surgery duration, min & $167[127-231]$ & $190[146-254]$ & 0.147 \\
\hline Anesthesia duration, $\min$ & $263[236-314]$ & $270[231-357]$ & 0.579 \\
\hline VAS score in recovery room & $7[5-9]$ & $7[5-9]$ & 0.962 \\
\hline \multicolumn{4}{|l|}{ Cortisol, $\mu \mathrm{g} / \mathrm{dl}$} \\
\hline Baseline & $12.45[8.80-19.15]$ & $9.65[5.75-12.25]$ & 0.002 \\
\hline Intraoperative & $31.8[14.55-38.60]$ & $4.8[2.90-9.60]$ & $<0.001$ \\
\hline Recovery & $31.35[18.45-45.80]$ & $13.4[3.40-17.70]$ & $<0.001$ \\
\hline \multicolumn{4}{|l|}{ Epinephrine, pg/ml } \\
\hline Baseline & $39[0-62]$ & $23[0-41]$ & 0.085 \\
\hline Intraoperative & $65[25-170]$ & $0[0-53]$ & $<0.001$ \\
\hline Recovery & $127[57-215]$ & $57[0-98]$ & 0.004 \\
\hline \multicolumn{4}{|l|}{ Norepinephrine, $\mathrm{pg} / \mathrm{ml}$} \\
\hline Baseline & $278[138-480]$ & $361[202-502]$ & 0.410 \\
\hline Intraoperative & $341[150-597]$ & $136[90-266]$ & $<0.001$ \\
\hline Recovery & $434[199-811]$ & 311 [162-467] & 0.107 \\
\hline Delirium in PACU & $1(3.2 \%)$ & $3(7.5 \%)$ & 0.622 \\
\hline \multicolumn{4}{|l|}{ Delirium postoperative days } \\
\hline $1-3$ & $8(22.2 \%)$ & $6(15 \%)$ & 0.417 \\
\hline
\end{tabular}

Data are presented as median [25th to 75 th percentile] or number (\%). The tests used for group comparisons are the 2-sample $t$ test, Wilcoxon rank sum test, $\chi^{2}$ test or Fisher's exact test, as appropriate. VAS = Visual Analog Scale score for pain in the recovery room; PACU = postoperative care unit. Cortisol $(\mu \mathrm{g} /$ $\mathrm{dl})$, epinephrine $(\mathrm{pg} / \mathrm{ml})$, and norepinephrine $(\mathrm{pg} / \mathrm{ml})$ : serum levels at baseline = immediately prior to surgery, intraoperative $=4 \mathrm{~h}$ after surgical start, recovery $=2 \mathrm{~h}$ after surgery finish.

Because of the very broad range of delirium in previous studies, which have been heterogeneous with respect to the surgical population and agents, and the diurnal variation of cortisol, the sample size for this study was based on the difference in norepinephrine response between the gas and TIVA groups, which has been better characterized. For this purpose, we used the difference in serum norepinephrine levels between groups from the work of Marana et al. [17], which showed a $300 \mathrm{pg} / \mathrm{ml}$ difference between patients who received gas and those who received TIVA. With a sample size of 75 using a 2 -sided 0.05 t test, we would have a greater than $80 \%$ power to detect a difference of this magnitude.

\section{Results}

Table 1 compares the TIVA and gas groups on baseline clinical and demographic variables. The TIVA group was younger (73.48 vs. 76.33 years, $p=0.024)$, had a higher baseline MMSE score (29 vs. 27, $\mathrm{p}=0.029$ ), and lower ASA physical status indicating less comorbid disease $(p=0.021)$. Table 2 compares intraoperative and postoperative independent variables between the TIVA and gas groups. Median EBL was higher in the TIVA group (375 vs. $200 \mathrm{ml}, \mathrm{p}=0.029$ ); anesthesia and surgical times were similar in both groups. Median postoperative pain scores as measured by the visual analog scale score were similar in both groups in the recovery room. The cortisol level was higher in the gas group at all time points $(p=0.002, p<0.001, p<0.001)$. Serum epinephrine and norepinephrine were similar at baseline but lower in the TIVA group during and after surgery. Despite the fact that the TIVA group was younger and had better baseline cognition, the incidence of immediate PD was not 
Deiner et al.: Do Stress Markers and Anesthetic Technique Predict Delirium in the Elderly?

different between the two groups in the postoperative care unit (gas 3.2\% vs. TIVA 7.5\%, p= 0.622 ) or on the postoperative days (gas $22.2 \%$ vs. TIVA $15 \%$, $\mathrm{p}=0.417$ ).

Table 3 compares patients who did and did not develop PD on postoperative days 1-3. The recovery room level of norepinephrine $(p=0.03)$ was much higher in patients who developed PD, with no significant difference in intraoperative levels of norepinephrine, cortisol or epinephrine. There was a trend towards an association between lower baseline MMSE score $(p=0.056)$ and higher ASA physical status indicating a sicker patient $(p=0.083)$, and preexisting diabetes $(\mathrm{p}=0.156)$; these were included in the multivariate analysis. A larger proportion of patients who had delirium in the recovery room (22.2 vs. 3.4\%) went on to have delirium on the postoperative days, but this result was not significant $(p=0.084)$. Duration of surgery ( $p=0.097$ ) was marginally associated with development of PD, but the anesthetic group, EBL, type of surgery and anesthesia duration were not associated with delirium.

Table 4 describes the multivariable stepwise logistic regression which models the outcome of PD including variables with univariate association $(\mathrm{p}<0.2)$ and clinical significance. The predictors which remained in the model were diabetes, surgical duration and the postoperative level of norepinephrine. Diabetics had 4.51 times the odds of developing PD (95\% CI 1.00-20.14). For every 100-pg increase in serum norepinephrine the odds of PD increased by approximately $20 \%$ (95\% CI 1.01-1.42). Surgical duration was a marginal predictor; for every 1-hour increase the odds of PD increased by 1.46 times (95\% CI 0.922.32). In a model which included these three variables, serum glucose, postoperative care unit delirium, surgical procedure, ASA classification, EBL and MMSE score were not significant. The area under the receiver operating characteristic curve for this model was 0.772 (fig. 1).

\section{Discussion}

In this study, there was no significant difference in the incidence of PD between elderly patients who received TIVA and those who received gas; however, the TIVA group demonstrated suppression of norepinephrine, epinephrine and cortisol during surgery. The recovery room level of serum norepinephrine was predictive of delirium and the median recovery room norepinephrine levels of patients who became delirious were higher than during surgery in any group. This suggests that the magnitude of the stress response, which may be quite high in the recovery room due to hypothermia, anxiety and pain, is more important in the development of PD than the anesthetic technique.

Our finding of the relationship between stress and delirium may have particular relevance for the elderly. Animal studies suggest that increased age is associated with prolonged stress response to systemic inflammation and longer-lasting cognitive impairment [21, 22]. Mechanistically, serum norepinephrine is associated with neuronal injury and worsened delirium, and prior studies suggest that $\alpha$-adrenergic blocking sedatives (dexmedetomidine) may decrease delirium in the intensive care unit $[1,23,24]$. In our study, serum epinephrine and cortisol levels were not different between patients who did and did not develop PD. These results are consistent with other studies which show that the magnitudes of the cortisol, epinephrine and norepinephrine response are not entirely parallel [17].

While the recovery room level of the serum norepinephrine response was important in our model, it was not the sole determining factor for the development of PD. Our multivariable model suggests that PD is the result of a more complicated interaction between stress and the underlying patient characteristic of diabetes. This suggests that preexisting vulnerability (e.g. diabetes) predisposes certain patients to delirium. The impact of diabetes is much broader than its effect on glycemic control; in our study, we did examine serum glucose (not shown) 
Table 3. Comparison of patients who did (PD+) and did not (PD-) develop delirium

\begin{tabular}{|c|c|c|c|}
\hline & PD- $(n=62)$ & $P D+(n=14)$ & $\mathrm{p}$ value \\
\hline Age, years & $73.48 \pm 5.03$ & $75.83 \pm 5.41$ & \\
\hline \multicolumn{4}{|l|}{ Gender } \\
\hline Male & $30(48.4 \%)$ & $10(71.4 \%)$ & \multirow[t]{2}{*}{0.146} \\
\hline Female & $32(51.6 \%)$ & $4(28.6 \%)$ & \\
\hline \multicolumn{4}{|l|}{ ASA status } \\
\hline 1 & $0(0 \%)$ & $1(7.1 \%)$ & \multirow{4}{*}{0.083} \\
\hline 2 & $17(27.4 \%)$ & $1(7.1 \%)$ & \\
\hline 3 & $37(59.7 \%)$ & $10(71.4 \%)$ & \\
\hline 4 & $8(12.9 \%)$ & $2(14.3 \%)$ & \\
\hline CAD & $8(11.9 \%)$ & $4(28.6 \%)$ & 0.217 \\
\hline CVA & $4(6.5 \%)$ & $1(7.1 \%)$ & 1.000 \\
\hline Diabetes & $11(17.7 \%)$ & $5(35.7 \%)$ & 0.156 \\
\hline \multicolumn{4}{|l|}{ Glucose, mg/dl } \\
\hline Baseline & $94[85-106]$ & $103[81-118]$ & 0.439 \\
\hline Intraoperative & $116[96-142]$ & $143[101-152]$ & 0.121 \\
\hline Recovery & $127[107-149]$ & $167[117-187]$ & 0.055 \\
\hline Malignancy & $30(49.2 \%)$ & $6(42.9 \%)$ & 0.771 \\
\hline Renal disease & $6(9.7 \%)$ & $1(7.1 \%)$ & 1.000 \\
\hline \multicolumn{4}{|l|}{ Anesthesia } \\
\hline Gas & $28(77.8 \%)$ & $8(22.2 \%)$ & \multirow[t]{2}{*}{0.417} \\
\hline TIVA & $34(85 \%)$ & $6(15 \%)$ & \\
\hline \multicolumn{4}{|l|}{ Surgery } \\
\hline Spine & $31(50 \%)$ & $6(42.9 \%)$ & \multirow[t]{4}{*}{0.621} \\
\hline Urologic & $8(12.9 \%)$ & $1(7.1 \%)$ & \\
\hline General & $16(25.8 \%)$ & $6(42.9 \%)$ & \\
\hline Thoracic & $7(11.3 \%)$ & $1(7.1 \%)$ & \\
\hline Baseline MMSE score & $29[27-30]$ & $27[25-29]$ & 0.056 \\
\hline $\mathrm{EBL}, \mathrm{ml}$ & $250[100-600]$ & $200[150-700]$ & 0.761 \\
\hline Surgery duration, min & $171[136-246]$ & $191[168-239]$ & 0.097 \\
\hline Anesthesia duration, min & $266[230-316]$ & $288[256-359]$ & 0.119 \\
\hline VAS score in recovery room & $7[5-9]$ & $7[6-10]$ & 0.350 \\
\hline \multicolumn{4}{|l|}{ Cortisol, $\mu \mathrm{g} / \mathrm{dl}$} \\
\hline Baseline & $11.25[8.1-13.9]$ & $9.3[5.3-11.7]$ & 0.178 \\
\hline Intraoperative & $13.6[4.8-32.3]$ & $10.5[3.3-31.3]$ & 0.740 \\
\hline Recovery & $18.8[8.3-33.2]$ & $14.6[12.6-31.7]$ & 0.978 \\
\hline \multicolumn{4}{|l|}{ Epinephrine, pg/ml } \\
\hline Baseline & $26[0-54]$ & $14.5[0-50]$ & 0.746 \\
\hline Intraoperative & $35[0-78]$ & $36[0-170]$ & 0.588 \\
\hline Recovery & 76 [39-145] & $131[48-196]$ & 0.388 \\
\hline \multicolumn{4}{|l|}{ Norepinephrine, $\mathrm{pg} / \mathrm{ml}$} \\
\hline Baseline & $296[174-480]$ & 419 [278-495] & 0.556 \\
\hline Intraoperative & $185[109-341]$ & $300[68-597]$ & 0.314 \\
\hline Recovery & $322[150-541]$ & $586[346-811]$ & 0.030 \\
\hline PACU delirium & $2(3.4 \%)$ & $2(22.2 \%)$ & 0.084 \\
\hline
\end{tabular}

Data are presented as mean \pm SD, median [25th to 75th percentile] or number (\%). The tests used for group comparisons are the 2 -sample t test, Wilcoxon rank sum test, $\chi^{2}$ test or Fisher's exact test, as appropriate. $\mathrm{CAD}=$ Coronary artery disease; $\mathrm{CVA}=$ cerebrovascular accident; VAS = Visual Analog Scale score for pain in the recovery room; PACU = postoperative care unit. Cortisol $(\mu \mathrm{g} / \mathrm{dl})$, epinephrine $(\mathrm{pg} / \mathrm{ml})$, norepinephrine $(\mathrm{pg} / \mathrm{ml})$, glucose $(\mathrm{mg} / \mathrm{dl})$ : serum levels at baseline $=$ immediately prior to surgery, intraoperative $=4 \mathrm{~h}$ after surgical start, recovery $=2 \mathrm{~h}$ after surgery finish. 
Dementia

and Geriatric

Cognitive Disorders

Table 4. Multivariable logistic model of delirium

\begin{tabular}{|c|c|c|c|}
\hline \multicolumn{4}{|c|}{ Dement Geriatr Cogn Disord 2014;38:366-374 } \\
\hline \multicolumn{2}{|l|}{ DOI: $10.1159 / 000363762$} & \multicolumn{2}{|c|}{$\begin{array}{l}\text { (c) } 2014 \text { S. Karger AG, Basel } \\
\text { www.karger.com/dem }\end{array}$} \\
\hline \multicolumn{4}{|c|}{$\begin{array}{l}\text { Deiner et al.: Do Stress Markers and Anesthetic Technique Predict Delirium in the } \\
\text { Elderly? }\end{array}$} \\
\hline Predictor & Unit & Odds ratio & $95 \% \mathrm{CI}$ \\
\hline $\begin{array}{l}\text { Surgical duration } \\
\text { Recovery room }\end{array}$ & $1 \mathrm{~h}$ & 1.46 & $0.92-2.32$ \\
\hline norepinephrine level & $100 \mathrm{pg} / \mathrm{ml}$ & 1.20 & $1.01-1.42$ \\
\hline Diabetes & $1=$ yes & 4.51 & $1.01-20.14$ \\
\hline
\end{tabular}

Fig. 1. Receiver operating characteristic (ROC) curve for this model to predict PD; the model includes recovery room level of serum norepinephrine, surgical duration, and diabetes. The area under the curve is 0.772 .

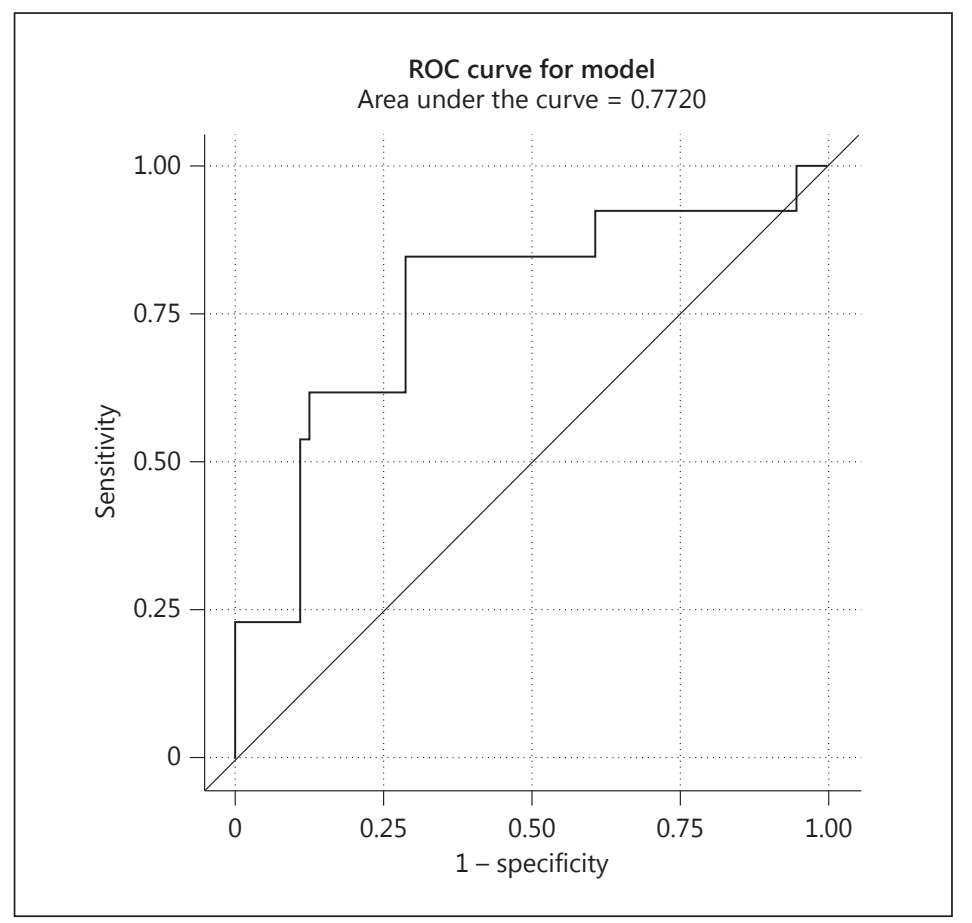

which was mildly higher in patients with PD but was not an independent predictor of PD. One possible mechanism by which diabetes may contribute to delirium aside from acute glycemic control includes its effects on the blood-brain barrier [25]. Previous studies have shown that the blood-brain barrier exhibits structural and functional changes with age and pathological conditions, e.g. type II diabetes [26, 27]. Systemic mediators such as serum norepinephrine have been shown to indirectly influence the central nervous system milieu by activating endothelial cells of the brain's vasculature to secrete prostaglandins or stimulate afferents of the vagus nerve which stimulate the nucleus tractus solitarii $[24,28]$.

In both the TIVA and gas groups, the incidence of delirium that we found was within the range described for older general surgery patients. The magnitude of the difference between groups is similar to that observed by Royce et al. but much larger than that found by T. Monk et al. in elderly patients having hip surgery [29]. Because this was not a randomized study, there were some important differences between the TIVA and gas groups which may have influenced this, including the fact that the TIVA group was approximately 3 years younger, had a somewhat higher baseline MMSE score, and had a lower ASA physical status (less comorbidity burden) than the gas group. These factors also seem potential driving factors for the outcome of delirium. However, in the multivariable model adjusted for the presence of diabetes, surgical duration and serum norepinephrine in the recovery room, the baseline features of age, MMSE score and ASA physical status were not significant. 
Deiner et al.: Do Stress Markers and Anesthetic Technique Predict Delirium in the Elderly?

This study has several limitations. It was underpowered to observe a difference in the incidence of delirium between the TIVA and gas groups. Based on the observed $7 \%$ difference between the groups, we would have needed 953 patients ( 476 in each arm) in order to achieve statistical significance. Whether this difference is clinically meaningful and worth further prospective study is questionable. The anesthetic was standardized but the patients were not randomized to either TIVA or gas because many patients were restricted to a group based on the surgery. For example, patients undergoing spine surgery often require neuromonitoring which is facilitated by the use of TIVA. However, there was no difference in the distribution of surgical procedures between patients who did and did not develop delirium.

While there were some baseline differences between the group (age, ASA status) we were able to measure and adjust for these. We also adjusted for surgical procedure, EBL and comorbidity.

\section{Conclusion}

In this study, we did not observe a clear association between the anesthetic technique and either emergence delirium or PD. We have found that the recovery room level of norepinephrine is much higher than that during surgery and is a risk factor for PD. Our findings suggest a complex interplay between the stress response in elderly patients and the underlying patient characteristic of diabetes. We hope that the presentation of these data will help refocus the study of PD to include the immediate postoperative period, which has not traditionally taken into consideration the geriatric patient and has several areas amenable to improvement (e.g., postoperative pain control, anxiety and temperature regulation). Given the evidence that delirium is associated with long-term cognitive sequelae and dementia, studies of the cognitive health and recovery of postsurgical elderly patients should focus on the postoperative period. Future studies should investigate the immediate postoperative period to identify strategies to prevent or mitigate PD.

\section{Acknowledgements}

This research is supported by NIH GEMSSTAR1 R03 AG040624-01, NIH P50 AG005138, NIH R01 AG029656-01A1, the American Geriatrics Society Jahnigen Scholar Program, and the Foundation for Anesthesia Education and Research (FAER).

\section{Disclosure Statement}

None of the authors have a conflict of interest related to the outcomes of this study.

\section{References}

1 Pandharipande PP, Pun BT, Herr DL, Maze M, Girard TD, Miller RR, et al: Effect of sedation with dexmedetomidine vs lorazepam on acute brain dysfunction in mechanically ventilated patients: the MENDS randomized controlled trial. JAMA 2007;298:2644-2653.

-2 Davis DH, Muniz Terrera G, Keage H, Rahkonen T, Oinas M, Matthews FE, et al: Delirium is a strong risk factor for dementia in the oldest-old: a population-based cohort study. Brain 2012;135:2809-2816.

-3 Witlox J, Eurelings LS, de Jonghe JF, Kalisvaart KJ, Eikelenboom P, van Gool WA: Delirium in elderly patients and the risk of postdischarge mortality, institutionalization, and dementia: a meta-analysis. JAMA 2010;304: 443-451.

4 Girard TD, Jackson JC, Pandharipande PP, Pun BT, Thompson JL, Shintani AK, et al: Delirium as a predictor of long-term cognitive impairment in survivors of critical illness. Crit Care Med 2010;38:1513-1520. 
Deiner et al.: Do Stress Markers and Anesthetic Technique Predict Delirium in the Elderly?

5 Verloo H, Goulet C, Morin D, von Gunten A: Delirium among home-dwelling elderly after a recent hospitalization: an urgent need for effective nursing interventions. Dement Geriatr Cogn Dis Extra 2012;2:187-189.

-6 Pitkala KH, Laurila JV, Strandberg TE, Tilvis RS: Prognostic significance of delirium in frail older people. Dement Geriatr Cogn Disord 2005;19:158-163.

7 Silverstein JH, Deiner SG: Perioperative delirium and its relationship to dementia. Prog Neuropsychopharmacol Biol Psychiatry 2013;43:108-115.

8 Deiner S, Silverstein JH: Postoperative delirium and cognitive dysfunction. Br J Anaesth 2009;103(suppl 1):i41-i46.

9 Franco K, Litaker D, Locala J, Bronson D: The cost of delirium in the surgical patient. Psychosomatics 2001;42: 68-73.

$>10$ Inouye SK: Delirium in older persons. N Engl J Med 2006;354:1157-1165.

11 Zhang H, Lu Y, Liu M, Zou Z, Wang L, Xu FY, et al: Strategies for prevention of postoperative delirium: a systematic review and meta-analysis of randomized trials. Crit Care 2013;17:R47.

12 Royse CF, Andrews DT, Newman SN, Stygall J, Williams Z, Pang J, et al: The influence of propofol or desflurane on postoperative cognitive dysfunction in patients undergoing coronary artery bypass surgery. Anaesthesia 2011;66:455-464.

13 MacLullich AMJ, Ferguson KJ, Miller T, de Rooij SEJA, Cunningham C: Unravelling the pathophysiology of delirium: a focus on the role of aberrant stress responses. J Psychosom Res 2008 9;65:229-238.

14 Hall RJ, Shenkin SD, Maclullich AM: A systematic literature review of cerebrospinal fluid biomarkers in delirium. Dement Geriatr Cogn Disord 2011;32:79-93.

15 Cerejeira J, Batista P, Nogueira V, Vaz-Serra A, Mukaetova-Ladinska EB: The stress response to surgery and postoperative delirium: evidence of hypothalamic-pituitary-adrenal axis hyperresponsiveness and decreased suppression of the GH/IGF-1 axis. J Geriatr Psychiatry Neurol 2013;26:185-194.

16 Roizen MF, Horrigan RW, Frazer BM: Anesthetic doses blocking adrenergic (stress) and cardiovascular responses to incision - MAC BAR. Anesthesiology 1981;54:390-398.

17 Marana E, Colicci S, Meo F, Marana R, Proietti R: Neuroendocrine stress response in gynecological laparoscopy: TIVA with propofol versus sevoflurane anesthesia. J Clin Anesth 2010;22:250-255.

18 Ledowski T, Bein B, Hanss R, Paris A, Fudickar W, Scholz J, et al: Neuroendocrine stress response and heart rate variability: a comparison of total intravenous versus balanced anesthesia. Anesth Analg 2005;101:17001705.

19 Inouye SK, van Dyck CH, Alessi CA, Balkin S, Siegal AP, Horwitz RI: Clarifying confusion: the confusion assessment method. A new method for detection of delirium. Ann Intern Med 1990;113:941-948.

-20 Folstein MF, Folstein SE, McHugh PR: 'Mini-mental state'. A practical method for grading the cognitive state of patients for the clinician. J Psychiatr Res 1975;12:189-198.

-21 Chapman TR, Barrientos RM, Ahrendsen JT, Maier SF, Patterson SL: Synaptic correlates of increased cognitive vulnerability with aging: peripheral immune challenge and aging interact to disrupt theta-burst late-phase long-term potentiation in hippocampal area CA1. J Neurosci 2010;30:7598-7603.

-22 Barrientos RM, Frank MG, Hein AM, Higgins EA, Watkins LR, Rudy JW, et al: Time course of hippocampal IL-1 beta and memory consolidation impairments in aging rats following peripheral infection. Brain Behav Immun 2009;23:46-54.

-23 Bhardwaj A, Brannan T, Martinez-Tica J, Weinberger J: Ischemia in the dorsal hippocampus is associated with acute extracellular release of dopamine and norepinephrine. J Neural Transm Gen Sect 1990;80:195-201.

24 Maldonado JR: Neuropathogenesis of delirium: review of current etiologic theories and common pathways. Am J Geriatr Psychiatry 2013;21:1190-1222.

25 Hughes CG, Morandi A, Girard TD, Riedel B, Thompson JL, Shintani AK, et al: Association between endothelial dysfunction and acute brain dysfunction during critical illness. Anesthesiology 2013;118:631-639.

-26 Mooradian AD: Potential mechanisms of the age-related changes in the blood-brain barrier. Neurobiol Aging 1994;15:751-755; discussion 761-762, 767.

-27 Starr JM, Wardlaw J, Ferguson K, MacLullich A, Deary IJ, Marshall I: Increased blood-brain barrier permeability in type II diabetes demonstrated by gadolinium magnetic resonance imaging. J Neurol Neurosurg Psychiatry 2003;74:70-76.

28 Uchikado H, Akiyama H, Kondo H, Ikeda K, Tsuchiya K, Kato M, et al: Activation of vascular endothelial cells and perivascular cells by systemic inflammation - an immunohistochemical study of postmortem human brain tissues. Acta Neuropathol 2004;107:341-351.

29 Study finds that anesthetics do not cause postoperative delirium in the elderly. 2011. http://www.asahq.org/ Home/For\%20the\%20Public\%20and\%20Media/Press\%20Room/Anesthesiology\%20and\%200ther\%20 Scientific\%20Press\%20Releases/Study\%20Finds\%20That\%20Anesthetics\%20Do\%20Not\%20Cause\%20 Postoperative\%20Delirium\%20in\%20the\%20Elderly.aspx. 